\title{
De la investigación mediante relatos de vida al teatro social: el caso de la brecha digital de género*
}

\author{
From Research Using Life Stories to Social \\ Theatre: The Gender Digital Divide Case
}

Recibido: 30 de octubre de 2014 | Aceptado: 02 de septiembre de 2015

\author{
ADRIANA GIL-JUÁREZ** \\ Universitat Rovira i Virgili, España \\ JOEL FELIU *** \\ Universitat Autònoma de Barcelona, España \\ MONTSE VALL-LLOVERA ***** \\ Universitat Oberta de Catalunya, España \\ Andrea CALSAMIGLIA ****** \\ Universitat Autònoma de Barcelona y Compañía de Teatro \\ NUS, España \\ ESTER CONESA ******* \\ Universitat Rovira i Virgili, España
}

doi : 10.11144/Javeriana.upsy14-5.imrv

Para citar este artículo:Gil-Juárez, A., Feliu, J., Valllovera, M., Calsamiglia, A., \& Conesa, E. (2015). De la investigación mediante relatos de vida al teatro social: el caso de la brecha digital de género. Universitas Psychologica, 14(4), 1583-1598. http:// dx.doi.org/10.11144/Javeriana.upsy14-5.imrv

* Artículo de investigación, resultado del proyecto "Trayectorias de vida tecnológica y género: factores psicosociales implicados en el acceso a las titulaciones de ingeniería informática" (código 96/10), financiado por el Instituto Nacional de la Mujer (Ministerio Español de Sanidad, Política Social e Igualdad), realizado de Octubre del 2010 a Octubre del 2013.

** Departament de Psicologia, edifici W043007, Tarragona.Correo electrónico: adriana.gil@urv. cat

*** Departament de Psicologia Social, edifici B 08193, Bellaterra (Cerdanyola del Vallès). Correo electrónico: joel.feliu@uab.cat

****** Estudis de Psicologia i Ciències de l'Educació Rambla del Poblenou, 15608018, Barcelona. Correo electrónico: mvall-llovera@uoc.edu

****** Departament de Psicologia Social, edifici B 08193, Bellaterra (Cerdanyola del Vallès). Correo electrónico: andrea.calsamiglia@uab.cat

******** Departament de Psicologia, edifici W043007, Tarragona. Correo electrónico: ester.conesac@ urv.cat

\section{RESUMEN}

El presente es un proyecto de investigación-acción, cuyo objetivo fue promover el interés por el uso experto de tecnologías en mujeres jóvenes, con miras a reducir la dominación masculina del sector tecnológico. A partir de la elaboración de relatos de vida de mujeres estudiantes de informática, diseñamos y representamos una obra de teatro-foro en institutos de secundaria, cuyo contenido recreaba las vivencias de las chicas con la tecnología. Las situaciones conflictivas se transformaron, con el fin de superar los problemas planteados y de empoderar a las participantes. Utilizando el teatro social, mostramos cómo se pueden ensayar nuevas formas de acción ante situaciones de opresión, criticamos las asociaciones establecidas entre género y tecnología y formulamos recomendaciones para favorecer que las mujeres opten por el ámbito tecnológico.

Palabras Clave

brecha digital de género; teatro social; investigación-acción; relatos de vida

\footnotetext{
A B S T R A C T

We present an Action Research program whose aim was to promote the skilful use of technology in young women to reduce the male dominance found in this sector. From Arising out of the elaboration of life stories of women studying computer science, we designed and performed a social theatre play in high schools that recreated the experiences of girls with technology. The conflicting situations were transformed in order to overcome the problems presented and to empower participating girls. We show how to test new forms of acting using Social Theatre in front of oppressing situations; we criticise the current associations between gender and technology; and, we formulate recommendations to encourage women to opt for ta technological career path.

Keywords

gender digital divide; social theatre; action research; life stories
} 


\section{Introducción}

Aunque, en los países occidentales, mujeres y hombres acceden a las Tecnologías de la Información y Comunicación (TIC) y las usan con la misma intensidad, las mujeres de estos países no han ido incorporándose en mayor número a los estudios de informática. Además, en las últimas décadas su presencia ha ido haciéndose cada vez menor (Gómez Ferri, 2004; Pérez -Fuentes \& Andino, 2003; Sanz, 2005, 2008; Black, Jameson, Komoss, Meehan, \& Numerico, 2005; Burrelli, 2008; Camp, Miller, \& Davies, 2000; Carlson 2006; Galpin, 2002). En otras palabras, mientras la primera brecha digital de género - la de acceso y uso- se cerraba, la segunda brecha - relativa a la experticia — se amplió (Castaño, 2008). Como afirma Hayes (2010) —refiriéndose a los Estados Unidos_-, "Si esta tendencia siguiera al ritmo experimentado de 1986 a 2006, no quedarían mujeres graduadas en informática en 2032" (p. 27).

En España, según el último informe sobre la Situación de la e-igualdad en los años 2012-2013 (Instituto de la Mujer y para la Igualdad de Oportunidades, 2015), la presencia de mujeres en el sector TIC, específicamente, en las ramas de contenidos y edición, alcanza el $45 \%$ y el $50.7 \%$ respectivamente. Sin embargo, su escasa presencia en las ramas de tecnología, telecomunicaciones y programación-30.6\%, 34.8\% y $26.5 \%$ respectivamentesugiere que, en el nivel experto, las mujeres siguen estando infrarrepresentadas.

En el contexto de las universidades del estado español, los estudios realizados por el Ministerio de Educación, Cultura y Deporte sobre oferta, demanda y matriculación, desde el curso 2003/2004 hasta el 2010/2011, constatan una disminución generalizada de la demanda y de las matrículas en las titulaciones técnicas en España. Más específicamente, a partir de los datos facilitados por el Instituto Nacional de Estadística, el descenso es significativamente apreciable en las matriculaciones y en el número de graduadas en Ingeniería Informática (Garzas, 2010). En esta carrera y en el curso académico 2003/2004, un 23\% del total de estudiantes matriculados eran mujeres, mientras que en el curso 2010/2011 descendió hasta el 15\%, según información provista en la página web del Instituto Nacional de Estadística de España. Según el informe "Científicas en cifras 2013" (Unidad de Mujeres y Ciencia, 2014), aunque la brecha digital de género ha experimentado una pequeña disminución, las mujeres siguen estando infrarrepresentadas en los campos de estudio tecnológicos, lo que alerta sobre la necesidad de tomar medidas y llevar a cabo acciones para resolver el problema.

A pesar de que el informe publicado por la $\mathrm{Co}-$ misión Europea (European Union, 2013) asegura que acercar a más chicas a carreras y puestos de trabajo tecnológicos es uno de los objetivos primordiales para la economía de muchos países, es importante anotar que, más allá de un problema económico o estratégico, se trata de un problema político que debe ser abordado desde una perspectiva feminista o mejor, tecnofeminista (Wajcman, 2006). La persistencia de la asociación entre tecnología y masculinidad daña las posibilidades de las mujeres. Si las mujeres ${ }^{1}$ no crean código, objetos virtuales, aplicaciones para móviles, programas y tampoco diseñan sistemas informáticos, se puede deducir que están teniendo lugar —al menoscuatro opresiones graves: en primer lugar, una dependencia tecnológica de las mujeres respecto de los hombres con los que conviven o trabajan; en segundo lugar, una vulnerabilidad que acaece cuando son agredidas en la red o cuando su derecho a la privacidad se ve atacado; en tercer lugar, una invisibilización de su contribución al desarrollo de la informática y el Internet y, en cuarto lugar, su exclusión de las innovaciones y creaciones que se realizan. En resumen, la ausencia de diversidad en

1 En este trabajo partimos de la propuesta de West y Zimmerman (1987) cuyo concepto doing gender permite describir cómo el género se actúa —-se performa—, se muestra y se despliega en las diferentes situaciones sociales. Es en ese 'hacer el género' que se reproducen las categorías sexuales — mujer y hombre- existentes en el discurso de género binario. Como toda actuación (performance), esta solo puede ocurrir en escenarios específicos. Visto así, el género es reproducido, negociado y resistido en la relación con la tecnología, y no algo que determine cómo es esa relación para cada categoría sexual. Cuando hablamos de 'mujeres', debe entenderse que hablamos de personas que, en general, actúan o responden como miembros de esa categoría sexual y, en este caso, en las interacciones con las investigadoras. 
el ámbito de las expertas en informática supone la exclusión social de amplias capas de la población — hombres y mujeres_- que no ven sus necesidades tenidas en cuenta (Heeter, Egidio, Mishra, Winn, \& Winn, 2008).

El estudio de la segunda brecha digital de género, que se relaciona con el uso experto de las TIC, se ha enfocado hasta ahora en la búsqueda de factores que expliquen la escasa presencia de mujeres en el mundo profesional de las tecnologías de la información, haciendo énfasis en la desafección que chicas y mujeres jóvenes sienten hacia las tecnologías y en los factores implicados en el rechazo o en la no opción por las carreras asociadas a las TIC (Margolis \& Fisher, 2002). Una metáfora dominante en este campo ha sido la de la "cañería que pierde" (Camp et al., 2000; Bartol \& Aspray, 2006; Departamento de Ciencia Política y Derecho Público, 2013 ; Vitores \& Gil-Juárez, 2015), según la cual las mujeres interesadas en la tecnología desaparecen en algún punto del recorrido. Sin embargo, a esta metáfora se le deben hacer dos importantes críticas: la primera, que la investigación inspirada por ella se ha focalizado únicamente en los factores que alejan a las mujeres de la informática, asumiendo que anular estos factores las incluirá (Soe y Yakura, 2008); la segunda, la escasa producción científica que ha generado en torno a los factores que inciden directamente en la inclusión, es decir, lo poco que se ha investigado aquello que influye a que se opte por estudiar, trabajar y disfrutar con esta actividad o profesión (Faulkner \& Lie, 2007; Lagesen, 2008; Dee, Petrie, Boyle, \& Pau, 2009; Vigdor, 2011; Vergés, Cruells, \& Hache, 2009; Vergés, 2012). Vergés (2012) estudia los procesos de autoinclusión de las mujeres en las TIC y apunta las posibilidades que se abren con la intersección de las tecnologías con otros ámbitos de conocimiento y actividad, como el arte y el activismo — ruta que queda excluida en la metáfora de la "cañería que pierde"- Las dos críticas permiten cuestionar la secuencia de pasos - ininterrumpida y unidireccional— sugerida por la imagen de la cañería, cuando presupone que el paso por cada etapa ocurre en el vacío social y cuando establece que el modelo de trayectoria profesional para las mujeres tiene que ser equivalente al modelo típicamente descrito para los hombres (Bartol \& Aspray, 2006; Castaño \& Webster, 2011; Corneliussen, 2011; Husband \& Myers, 2012; Jesse, 2008; Leventman, 2007; Soe \& Yakura, 2008). Esto invisibiliza a las mujeres que ya desarrollan actividades en el sector tecnológico (Faulkner \& Lie, 2007). Otros estudios relacionan la brecha digital de género con la no-inclusión en distintas formas de ocio tecnológico (Beavis \& Charles, 2007; Jenson \& de Castell, 2010; Kafai, 1998, Gil-Juárez, Feliu, \& Vitores, 2010). En consecuencia, cualquier intervención que tenga por objeto incorporar perfiles diversos al campo tecnológico necesita, primero, conocer las trayectorias vitales, de ocio y profesionales de quienes acceden al campo tecnológico.

También debe tenerse en cuenta que, como afirma Judy Wajcman (1991), el género y la tecnología no son entidades separadas sino mutuamente constituidas. El uso, el diseño, el desarrollo y la implementación de la tecnología juegan un papel en la propia conformación de los géneros (Jenson \& de Castell, 2008, 2010). También Gil-Juárez, Feliu, Vitores y Vall-llovera (2011) afirman que actualmente el tipo de performación que se requiere del género femenino es discordante con lo que implica actuar como experta en TIC, la cual se corresponde con la performación del género masculino. En consecuencia, resulta fundamental conocer las dificultades que se presentan al querer performar simultáneamente el género femenino y la experticia en tecnología (Gil-Juárez et al., 2010), pero también explorar las posibilidades de transformación que supone el hecho de hacerlo - de performar ambos a la vez-. Además, es importante que las personas interesadas en la tecnología tengan acceso a modelos con los que puedan identificarse (Lockwood, 2006).

Teniendo esto presente, quisimos plantear un proyecto de investigación-acción que contribuyera a reducir la dominación masculina en el sector tecnológico. Diferentes mujeres jóvenes estudiantes de informática nos relataron su trayectoria personal y su relación con la tecnología; a partir de estos relatos, diseñamos, representamos y evaluamos una obra de teatro-foro que recreaba las vivencias de estas mujeres con la tecnología. Mediante técnicas 
del teatro social, las situaciones se transformaron para superar los problemas planteados en la obra y empoderar a las chicas participantes.

La investigación y la intervención subsiguientes partieron de dos objetivos. Primero, obtener un conocimiento localmente significativo que posibilitara visibilizar las trayectorias de vida tecnológica de estudiantes universitarias de ingenierías informáticas y, en segundo lugar, atraer la atención de otras mujeres más jóvenes hacia el uso experto de TIC e incentivar su deseo por estudiar ingenierías informáticas, sin querer esconder, con ello, las dificultades de orden social con las que se pueden encontrar. Específicamente, buscamos identificar las visiones, relaciones y apropiaciones de las TIC de las participantes, analizar las experiencias, percepciones y valoraciones que hacen del ocio tecnológico y conocer sus sentimientos de competencia tecnológica. Lo anterior con la finalidad de crear, implementar y evaluar un proyecto de intervención empoderador mediante el cual hacer visibles las experiencias de las jóvenes entrevistadas, además de mostrar distintas situaciones en relación con la performación del género y la tecnología y generar espacios donde emerjan posibilidades de performaciones alternativas en la relación con la tecnología. Para ello, recurrimos al teatro social o Teatro del Oprimido (Howard, 2004; Puga Rayo, 2012).

\section{Metodología}

Atendiendo a los objetivos, optamos por una variante de investigación-acción que guiara el desarrollo y la aplicación de una intervención teatral basada en una investigación cualitativa. Las técnicas cualitativas, y sus fundamentos teóricos y epistemológicos, las convierten en privilegiadas para investigar las experiencias, los procesos y las relaciones humanas desde una vertiente comprensiva e interpretativa, las experiencias, los procesos y las relaciones de las personas (Denzin \& Lincoln, 2000). Construimos, a partir de las entrevistas, relatos de vida (Bertaux, 2005; Pujadas, 1992) que nos permitieron conocer sus trayectorias vitales y analizar los vínculos entre su vivencia, uso y tipo de relación con las tecnologías durante la infancia y adolescencia y la elección de estudiar carreras de perfil técnico.

\section{Muestra en la fase de investigación}

Utilizamos una muestra de tipo propositivo (Schutt, 2001), para la cual establecimos tres criterios de selección: (1) que fueran personas que se identificaran a sí mismas como mujeres, (2) que cursaran un grado en ingeniería informática y (3) que lo hubieran empezado recientemente con el fin de que tuvieran próximo el relato de los motivos por los que escogieron su carrera-. Para llegar a las estudiantes, hicimos un primer contacto a través de conocidos, siguiendo luego una táctica muestral de 'bola de nieve'2. Por otro lado - y dada la dificultad que suponía el escaso tamaño de la población - contactamos a las mujeres enviando correos electrónicos a los directores y directoras académicas de los centros de estudio. Siguiendo los criterios de completitud y saturación (Rubin \& Rubin, 1995), entrevistamos a trece chicas —once de ellas entre los 18 y 24 años, una de más de 30 años y otra mayor de 40_, siete de las cuales cursaban primer semestre de ingeniería informática y cinco de ellas segundo semestre. Las chicas estudiaban en seis universidades catalanas; una de ellas cursaba sus estudios de Ingeniería Informática al tiempo que los de Ingeniería en Sistemas Audiovisuales.

\section{Procedimiento de obtención de los relatos de vida}

La técnica usada habitualmente para producir relatos de vida es la entrevista semiestructurada (Miller, 2004). Elaboramos un primer guion y luego lo pusimos a prueba en tres entrevistas — que no formaron parte de la muestra final-, a partir de las cuales establecimos el guion definitivo. La pregunta inicial

2 "El muestreo en "bola de nieve" es especialmente útil en poblaciones escasas como la que nos ocupa y consiste en utilizar a los propios participantes para reclutar a otros que cumplan las mismas condiciones. 
fue por el primer recuerdo de un contacto con un ordenador o con una consola de videojuegos. De allí se desplegó una conversación sobre los usos que le dieron al ordenador o a la consola durante la infancia y adolescencia - tiempos, espacios y personas implicadas-, cómo aprendieron a usarlos, el contexto tecnológico familiar, escolar y de sus amistades, las razones por las cuales decidieron estudiar un grado en informática, sus vivencias iniciales en la carrera y, para acabar, sus visiones sobre su futuro. Durante el 2012, se llevaron a cabo las entrevistas en lugares, días y horas acordados con cada una de las estudiantes. Fueron informadas previamente acerca del doble objetivo del estudio y se les solicitó un consentimiento informado. Se les comunicó que mantendríamos su identidad en la confidencialidad, que sus palabras podrían ser citadas en diversos productos procedentes del estudio, que podían preguntar cualquier cosa sobre su papel en la investigación y que podían no responder a las preguntas cuando no quisieran hacerlo y abandonar la entrevista cuando así lo quisieran. Las entrevistas fueron grabadas íntegramente y transcritas para su análisis posterior.

\section{Análisis de las entrevistas}

Efectuamos un análisis de contenido de las entrevistas, con la finalidad de disponer de información manejable que nos permitiera ordenar los relatos y acercarnos a las trayectorias vitales de las chicas entrevistadas y a la comprensión de las situaciones vividas con las TIC, sus posicionamientos, afectos y decisiones. Lo hicimos de manera sistemática, a través de las cinco fases que proponen Strauss y Corbin (1998): segmentación de los datos; codificación de apertura; clasificación e interrelación de códigos; codificación focalizada, y (re)contextualización en el marco de los objetivos de la investigación.

\section{Intervención teatral}

El formato de investigación-acción que escogimos fue un teatro-foro: una estrategia que se encuentra en el Teatro del Oprimido (Boal, 2002, 2006). Nuestro proceso presenta algunas pequeñas dife- rencias respecto a la investigación-acción clásica, para la cual debe existir una 'comunidad' o 'grupo' oprimido - bien delimitado — con el cual se define conjuntamente el problema, se trabaja el empoderamiento y se co-construye el conocimiento y la acción. En nuestro caso, fue a partir de los aportes de las mujeres entrevistadas que las investigadoras identificamos los puntos problemáticos o conflictivos que pudieron alejarlas de la tecnología, para luego convertirlas, con el apoyo de profesionales del grupo de Teatro NUS, en escenas de una obra de teatro-foro. El grupo con el que trabajamos fue una comunidad de mujeres jóvenes que vieron coartadas sus opciones vitales y laborales por ser la tecnología un espacio social de dominación masculina. La distancia entre las mujeres y la tecnología conduce a formas de opresión; de ahí que las herramientas ofrecidas a las participantes en el teatro-foro supongan un empoderamiento para ellas.

El teatro-foro es una obra de teatro donde la tensión entre el protagonista y el antagonista, a nivel microsocial, responde a una situación de desigualdad u opresión del contexto macrosocial. En este caso, las investigadoras fueron el puente entre las personas que participaron de la investigación y la compañía de teatro que creó la obra, contribuyendo a que la voz de las entrevistadas fuera incluida en el guion. Este último se basó parcialmente en el análisis de las entrevistas.

La obra muestra situaciones cotidianas relativas a la brecha digital de género. Durante la obra, la protagonista intenta hacer frente a estas situaciones, mientras que los y las antagonistas intentan perpetuar el status quo desigual. Esto ocurre, normalmente, ante la mirada de otro personaje que observa desde el silencio. Al final de la obra, la persona que dinamiza el foro pregunta al público por lo ocurrido e invita a las personas a subir a escena y transformar aquello que ellas consideren injusto. Durante el foro, la persona que dinamiza facilita la visibilización, el análisis y la transformación de las desigualdades a partir de situaciones cotidianas. Los micromachismos son expresiones del machismo normalizadas socialmente que suelen pasar desapercibidas (Bonino, 1995) que sufre la protagonista en relación con 
su orientación vocacional a nivel microsocial y se corresponden con la brecha digital de género a nivel macrosocial. Así, cuando una persona del público confronta a los y las antagonistas en el escenario, está asimismo confrontando el machismo del personaje, a la institución a la que representa — familia, escuela, etc. — y a la sociedad patriarcal en general. La persona que sube a escena está jugando con el poder, explorando sus mecanismos y ensayando un cambio, al tiempo que genera condiciones que le permiten imaginar soluciones diferentes y nuevas posibilidades. Aquí yace el potencial empoderador del teatro-foro.

Siguiendo a Montenegro (2011), empoderar es, para nosotros, "fortalecer a las personas de la comunidad (...) para que puedan adquirir grados crecientes de autonomía y logros cada vez mayores en la transformación de su realidad" (p. 128). Sin embargo, no nos referimos a una única comunidad 'afectada', sino al conjunto de chicas y mujeres que comparten la opresión del distanciamiento de la tecnología, establecida como parte de la performación del género femenino en las sociedades occidentales. Adoptamos una perspectiva situada que apuesta por un enfoque participativo en la intervención social (Montenegro, 2011) y que asume como prioritaria la incorporación de los diferentes puntos de vista de los y las agentes sociales implicadas en una situación. El eje central de esta perspectiva es la consideración de que la mirada y el conocimiento de los y las investigadoras e interventoras no son la única herramienta para definir y solucionar un problema social: los significados sociales se definen de manera conjunta y se espera que la obra de teatro permita que así ocurra.

\section{Muestra en la fase de intervención}

La obra fue presentada a 784 chicas y chicos entre los 14 y 19 años, quienes cursaban en ese momento tercer y cuarto año de secundaria obligatoria y primero de bachillerato. La obra fue creada por la compañía de teatro NUS y representada en diez institutos de educación secundaria (IES), en seis localidades de Cataluña, entre mayo y junio del 2013.

\section{Resultados}

En primer lugar, describimos algunos resultados de las entrevistas en las que nos basamos para construir las escenas de la obra de teatro, cuyos resultados comentaremos en segundo lugar.

Formas de relación con y alrededor de las TIC

Las entrevistadas hablan recurrentemente de la "curiosidad" que les produce el funcionamiento de todo aquello que les rodea y encuentran en ella un elemento fundamental que las define como científicas o ingenieras. Sin embargo, también señalan insistentemente que se interesan más por lo que ellas llaman "útil” y "aplicable", en otras palabras, por lo que ayuda a las personas en la vida práctica. Programar, trabajar con código o crear apps son para ellas instrumentos para un fin, y deben servir para que las vidas de las personas mejoren. Así las cosas, el desarrollo de tecnología es, para ellas, compatible con una forma de feminidad más tradicional, vinculada a los cuidados. Estas mujeres también se refieren a su paso por la carrera tecnológica como un camino difícil, que solo se debe iniciar si "realmente te gusta" y "de verdad quieres hacerlo". Aseguran que hay que estar preparada para esforzarse constantemente y que no es una decisión que se pueda tomar sin problemas - como sucede cuando se decide cursar carreras del ámbito de la salud, afines con la performación del género femenino-.

Todas las chicas cuentan con referentes e influencias que las han animado a realizar sus estudios informáticos — con frecuencia su madre, su padre, algún familiar o docente-. Estos referentes o influencias, en ocasiones, transmitieron a las mujeres un "afán de superación" y generaron, así, la suficiente confianza en ellas mismas y en sus capacidades.

En algunos casos, las madres de estas mujeres trabajan o trabajaron como informáticas. En otros, hubieran querido hacerlo pero no pudieron, dadas a las barreras de su tiempo. En ambos casos, lograron transmitirles su propio entusiasmo. Sin embargo, y en la mayoría de los casos, las madres de las chicas no tenían mucho interés por los ordenadores, pero 
las apoyaron y motivaron. Pero el apoyo explícito está acompañado, muchas veces, de acciones que pueden transmitir otro mensaje, por ejemplo, de presión para que se abandone determinada actividad asociada a la tecnología para vincularse a actividades de socialización más 'adecuadas' para su género.

\section{Las prácticas y usos en torno al ocio tecnológico}

Algunas de las chicas no juegan y no jugaron nunca con videojuegos. Sus primeros acercamientos a un ordenador fueron con frecuencia con fines lúdicos —navegando por Internet, por ejemplo—. Uno de los efectos de la performación del género femenino es la tendencia a considerar los videojuegos como una pérdida de tiempo o como una inmadurez (GilJuárez, Feliu, \& Vitores, 2010): quienes sí jugaron no lo hicieron tanto como sus compañeros hombres, cosa que se hizo más evidente en la adolescencia y en la actualidad, como afirma una de las entrevistadas:

¿Videojuegos? Sí, sí [ríe avergonzada]. Mucho. No, y todavía juego, pero no... o sea, en las vacaciones, no estos que cada día estás ahí... no. Cuando tengo tiempo libre que no tenga que estudiar ni hacer nada, si no quedo con los amigos, sí que a veces juego. Pero bueno, muy de vez en cuando. (Mireia (pseudónimo), comunicación personal, 15 de Junio de 2012)

Desde su infancia hasta el momento de la entrevista, estas chicas han construido su interés por el ocio tecnológico de formas muy distintas, desinteresándose e interesándose de nuevo según las circunstancias, las demandas del entorno y las posibilidades reales del tiempo disponible:

Ya no juego, prácticamente... o sea, ahora ya... a la que tengo tiempo libre normalmente quedo con los amigos (...) porque, si no, pierdo el contacto totalmente ya. Con lo poco que los veo, ya solo me falta que el tiempo libre lo dedique a jugar a videojuegos. (Mireia (pseudónimo), comunicación personal, 15 de Junio de 2012)
De esta manera, ellas reproducen el discurso de sus padres, de acuerdo con el cual las actividades de socialización son más importantes que aquellas asociadas a la tecnología.

\section{Sentimiento de competencia y enfrentamiento de las dificultades}

Con excepción de una de las entrevistadas, las demás no realizaron ninguna actividad de formación relativa a la tecnología previa a la carrera. Aunque alguna podía tener nociones muy básicas de programación, de programación web o algunos conocimientos elementales de hardware, una buena parte de sus compañeros hombres — según explican ellas mismas - sí entraron a la carrera sabiendo ya programar, fuera que lo hubieran aprendido de manera autodidacta o de sus amigos o porque habían tomado cursos previos de formación en informática. Ello situó a las chicas en clara desventaja, al menos inicialmente, respecto de sus compañeros hombres.

Otra dificultad que enfrentan las chicas se relaciona con la difusión popular de un discurso neurosexista (Fine, 2010), que sostiene que las diferencias en términos de rendimiento técnico y matemático son naturales, como comenta otra de las entrevistadas:

Leí hace poco que decía que un... bueno, estudios que estaban probados, que se supone que los hombres tenían (...) el hemisferio izquierdo del cerebro más desarrollado que el derecho y que se habían atribuido las cualidades en plan conducción, espacial, todo el tema así... como de matemáticas, de tecnología, (...) pues estaba más predispuesto a hacer este tipo de cosas, que no la mujer (...) Entonces que ya no es solo una cosa de gustos, sino que a lo mejor sí que es verdad, bueno, se supone que es verdad, porque como está probado científicamente, que lo han probado de verdad... que no es solo de que te guste o no te guste sino que tienen más facilidad los chicos para hacer este tipo de cosas que no las chicas (...) a una chica a lo mejor le cuesta más entender las matemáticas que no a un chico. (Núria (pseudónimo), comunicación personal, 31 de Julio de 2012) 
Como en el caso del efecto Pigmalión ${ }^{3}$, reproducir este discurso no puede sino generar efectos negativos en la percepción de las chicas sobre su propia competencia y capacidades. Las amistades juegan un papel muy importante en la adolescencia $\mathrm{y}$, a pesar de que no todas las entrevistadas relatan experiencias negativas - o no las exteriorizan como negativas-, algunas aseguran que el hecho de no salir a la calle o verse con sus amigas por estar acabando algo en el ordenador las marcó de un modo u otro. Incluso, algunas afirman que sacar buenas notas las convertía en las 'raras' de la clase. La 'rareza', en este caso, las constituye como personas diferentes - ilegítimas en cierto modo-, por tener aficiones distintas a las de otras mujeres como ellas o al no participar de los espacios normativos para el género que les fue asignado.

Asimismo, los profesores y profesoras de primaria y secundaria son un referente fundamental para las chicas. Ellos y ellas les transmitieron información sobre quiénes son ellas y cómo deberían actúan y performar su género. Una de las entrevistadas que hacía programación web antes de entrar en la carrera y la única que se mostraba especialmente interesada por el dominio de la tecnología - fue definida por sus profesores como "la niña que de niña no tiene nada”. El mensaje que ello transmite es que las chicas tienen que escoger entre interesarse por la tecnología y ser mujeres.

\section{Desarrollo de la intervención}

Las situaciones de discriminación identificadas en las entrevistas fueron dramatizadas e integradas a la obra por los profesionales del grupo de teatro, con la asesoría de las investigadoras. Los y las adolescentes identificaron los efectos de la opresión ejercida en las situaciones expuestas en la obra y propusieron estrategias alternativas para disminuirlos o eliminarlos. Esto, a su vez, permitió visualizar usos y prácticas positivas para la inclusión de las mujeres

3 El efecto Pigmalión se refiere a las mejoras en los resultados que consiguen los discentes cuando sus docentes tienen expectativas elevadas sobre su rendimiento. Fue descrito por primera vez por Robert Rosenthal y Lenore Jacobson en 1966 en el ámbito de la tecnología. En la representación, las interacciones conflictivas con la tecnología se hicieron evidentes en tres momentos: primero, en una escena familiar donde el padre, basándose en un discurso neurosexista y paternalista, impedía que la protagonista se orientara profesionalmente hacía la informática; luego, en una escena en la que un amigo le niega a la protagonista su colaboración para resolver algunas dudas sobre programación solo porque es una chica, y, por último, una escena escolar en la que el profesor duda que la protagonista pueda resolver un error técnico en el ordenador que él está usando.

La escena de la familia fue seleccionada en siete de las diez representaciones. En todos los casos se identificó una discriminación de género y se tildó al padre de machista. Entre las razones expuestas por el público para afirmar lo anterior, estuvieron las siguientes: el padre afirma que la tecnología es cosa de hombres; el padre desacredita a su hija; el padre no tiene confianza en las habilidades y aptitudes de las mujeres; el padre no valora a su hija como la debería valorar y, por último, el padre dice que el cerebro de los hombres es más desarrollado que el de las mujeres. Las y los participantes propusieron que la hija fuera más contundente con su padre; que la hija le enseñe a su padre las habilidades que tiene con la informática; que la chica responda al padre que "si quiere hacer un hijo a su manera mejor que tenga otro hijo"; que la mujer obvie a su padre y muestre seguridad en lo que quiere hacer; que busque datos empíricos que contrarresten el argumento del padre y que exponga las ventajas de cursar un bachillerato tecnológico. Otra alternativa propuesta por el público fue cambiar el rol de la pareja sentimental del padre. En este caso se proponía que apoyara los argumentos de la hija en contraposición al padre.

La escena relacionada con los amigos fue seleccionada en seis de las diez representaciones. Los momentos que el público identificó como problemáticos fueron cuando el amigo de la protagonista exclamó “iMujeres!" en un tono despreciativo, sugiriendo que las mujeres no saben cómo arreglar un problema informático, y cuando el amigo no ayudó a la protagonista a resolver el problema infor- 
mático que tenía. El público planteó las siguientes opciones: que la protagonista: le diga "i¿De qué vas?!", cuestionándole; que insista, ya sea de forma sutil o agresiva, que el amigo le explique cómo resolver el problema; que le diga al amigo que si le enseña cómo solucionarlo, ya no necesitará volver a explicárselo de nuevo y, por último, que le pida a una amiga que le dé apoyo.

La escena del profesor fue seleccionada en cuatro de las diez representaciones. El público entendió que se trataba de actitud machista y que el profesor estaba discriminando a la protagonista por ser mujer. Las propuestas alternativas a esta situación fueron que la protagonista demostrara sus habilidades y que sus compañeros de clase le dieran apoyo y credibilidad —especialmente el chico al que el profesor pidió ayuda-. El público propuso demostrarle al profesor que las mujeres sí pueden lidiar con los ordenadores por sí solas.

\section{Evaluación de la intervención}

Al finalizar la intervención, quisimos saber si los y las jóvenes habían aprendido algo de las representaciones teatrales creadas a partir de los fragmentos de los relatos de vida de mujeres que habían tenido una relación problemática con la tecnología. También quisimos saber si ello había cambiado su punto de vista sobre la relación entre las mujeres y la tecnología. Con esta finalidad, elaboramos un cuestionario de evaluación para el público. Obtuvimos respuestas de 315 de los y las jóvenes que asistieron a las obras - 145 chicos, 152 chicas y 18 otros $^{4}$ - cuyas edades variaban entre los 14 y 19 años - el 84\% entre los 14 y 16 años-.

Al preguntarles si habían aprendido y qué habían aprendido, la mayoría de los chicos y chicas (71.7\%) respondió que sí. La categorización de las preguntas abiertas nos permite afirmar que la mayoría (109 chicas y chicos) opinaba que había aprendido algo en relación con la brecha digital de

4 El cuestionario daba tres opciones para la pregunta 'sexo': femenino, masculino y otros. Esta última opción se incluyó para aquellas y aquellos que no se sintieran identificadas o identificados con ninguna de las dos primeras opciones.

\begin{tabular}{l|l|l} 
Universitas Psychologica & V. 14 & No. 5
\end{tabular} género, algo sobre la privacidad en Internet (62) o sobre ambas cosas (17). Así las cosas, más de la mitad (55.7\%) de los y las jóvenes aprendió algo sobre la brecha digital de género.

El análisis de contenido de las respuestas de quienes dijeron no haber cambiado su punto de vista puso de manifiesto que eran chicos que de antemano creían en la igualdad entre hombres y mujeres y, por extensión, creían que la informática no era solo para los hombres. Algunos manifestaron ser previamente conscientes de la discriminación de género y de la opinión generalizada de que las chicas no tienen capacidad para la tecnología.

En cambio, quienes afirmaron que su punto de vista sí había cambiado argumentaron que la obra los hizo conscientes de un problema que desconocían, pues no sabían que las chicas no solían escoger carreras tecnológicas. Dijeron creer, tras el aprendizaje, que las mujeres que quisieran estudiar una carrera tecnológica debían seguir adelante con ello y no renunciar. Además, afirmaron haberse dado cuenta de que las mujeres eran capaces de hacer más cosas con la tecnología de lo que creían y que, incluso, en algunos casos podrían ser mejores que los hombres y que debería haber más chicas en el mundo de la tecnología. Los chicos y las chicas sugirieron a otra chica participante que cursara el bachillerato tecnológico.

La intervención mostró que - al menos para un grupo de chicos y chicas - la puesta en escena de acciones alternativas, propuestas por los participantes respecto a situaciones problemáticas asociadas a la tecnología y en relación con la performación del género, provocó que aprendieran sobre ello, que se identificarán con la problemática y que tuvieran conciencia sobre la brecha digital existente.

\section{Un sitio web como herramienta complementaria}

Al finalizar el proyecto creamos un sitio web ${ }^{5}$, disponible en catalán y castellano y dirigido principalmente a chicas jóvenes. El sitio está constituido

5 Dirección de la url creada: http://joventic.uoc.edu/ 
por los fragmentos de los relatos de vida tecnológica de las mujeres que entrevistamos y de los vídeos de distintos momentos del teatro-foto. Con ello, quisimos hacer visibles las distintas rutas que las entrevistadas han seguido para llegar a sus estudios e incorporar las experiencias que viven en relación con el acceso a las tecnologías. Tales experiencias sugieren diferentes estrategias para enfrentar las situaciones problemáticas. Organizamos estas experiencias alrededor de los tres momentos cruciales de la obra de teatro, identificados por los y las jóvenes - la familia, las amistades y el profesorado-. A cada una de ellas le corresponde una escena de la obra original y las alternativas sugeridas por los diferentes públicos de los IES. Aunque el sitio se dirige principalmente a las chicas interesadas en la tecnología, contiene información para padres, madres y profesorado sobre cómo usar la página de forma didáctica.

\section{Discusión}

El análisis de los resultados obtenidos confirmó que no es posible equiparar las trayectorias femeninas y las masculinas sin establecer matices y que no hay una única secuencia, ininterrumpida y unidireccional, para llegar a los estudios informáticos, como lo sugiere la metáfora de la "cañería que pierde" (Bartol \& Aspray, 2006; Castaño \& Webster, 2011; Corneliussen, 2011; Husband \& Myers, 2012; Jesse, 2006; Leventman, 2007; Soe \& Yakura, 2008). La atención que pusimos en los momentos de la entrevista donde aparecía la relación de la entrevistada con la tecnología nos permitió ver una amplia diversidad de relaciones posibles, que no hubiéramos podido ver sin poner el foco en la interacciones específicas. La performación del experto en tecnología no se realiza únicamente mediante los patrones masculinos, asumidos como norma y como neutrales (Gil-Juárez et al., 2010). Existen muchas trayectorias para ser experta, demasiados accesos, interrupciones, salidas y recorridos como para considerar la "cañería que pierde" una metáfora útil. Los relatos de las dos chicas mayores que entrevistamos muestran que se puede entrar al mundo de la tecnología en distintos momentos y sitios — en un caso, después de una licenciatura en traducción y, en el otro, después de varios intentos con otras carreras y en la mitad de su vida profesional. Como describieron Bartol \& Aspray (2006) y Jesse (2006), existen rutas alternativas de acceso a las carreras tecnológicas, diferentes a la ruta prototípica que suelen realizar los hombres. Estos accesos 'otros' muestran que las chicas llegan al terreno de lo tecnológico por diferentes caminos y con experiencias muy diversas. En los relatos de vida que atestiguamos, encontramos que las motivaciones eran hacer algo útil y aplicado para los demás, asî como la voluntad de saber cómo funcionan las cosas, siendo la curiosidad un motor principal. Detrás de sus historias también hay madres y padres que empujan, videojugadoras y creadoras de páginas web. La literatura señala, además, los motivos políticos, como la participación en comunidades hackers o la llegada a la informática desde otras ocupaciones y profesiones, como la medicina, el arte, el cine, la animación, la química u otras ingenierías (Vergés, 2009; Vergés, 2012).

Estas otras trayectorias, que podríamos llamar rutas invisibles por cuanto no tienen reconocimiento social, no son 'carentes' con respecto a las trayectorias masculinas, sino distintas. Ello significa que quien no siga las rutas establecidas o de mayor reconocimiento puede, sin embargo, seguir una carrera tecnológica por otra ruta y desenvolverse satisfactoriamente en ella. Nombrando y señalando estas trayectorias es que las hacemos visibles y, quizá, facilitamos que otras chicas las consideren como un posible itinerario. En este sentido y a diferencia de lo que generalmente se hace en este campo de investigación, no tratamos de buscar a las mujeres que no se hubieran "perdido en la cañería" y que hubieran logrado seguir la ruta de los hombres (Soe $\&$ Yakura, 2008), sino de hacer visibles a las mujeres que han seguido rutas hasta ahora invisibles y comprender, desde sus relatos, las complejidades y dificultades que enfrentaron, pero también las cosas agradables del camino. Con ello, pudimos conocer los factores de inclusión, esto es: aquello que hace que se opte por estudiar, trabajar o disfrutar del ámbito tecnológico (Faulkner \& Lie, 2007; Lagesen, 2008; Dee et al., 2009; Vigdor, 2011; Vergés et al., 2009; Vergés, 2012). 
También pusimos de manifiesto que las trayectorias tecnológicas no ocurren en el vacío social y que, por ello, es importante hablar de las situaciones específicas que hacen a las personas expertas en tecnología, esto es, qué sienten, qué hacen y en qué momentos son reconocidas y ejercen como tales. Es empoderador reconocer y difundir las dificultades con las que las chicas entrevistadas se encuentran o se han encontrado, las prácticas que, a pesar de las dificultades, les permiten hacer lo que quieren y dedicarse a la tecnología y las estrategias que los y las adolescentes propusieron para sortear las situaciones que identificaron como problemáticas en la obra. Lo primero porque permite que los y las participantes piensen las dificultades de manera diferente y que estén dispuestas a discutirlas con quienes les rodean, sabiendo que todas y todos pueden hacer algo para cambiarlas. Segundo, porque se genera un espacio en el que pueden probar formas de afrontar y renegociar las situaciones conflictivas que tienen que ver con la tecnología. Y, tercero, porque la obra también empodera a las chicas y chicos que no tienen un interés manifiesto por la tecnología, al hacerles descubrir que también tienen un rol fundamental en el apoyo y la no-discriminación de las chicas que sí están interesadas. Finalmente, incluimos y visibilizamos las 'rutas invisibles' y las experiencias de las chicas - algunas de desigualdad, otras de voluntad y determinación - en nuestro sitio web, contribuyendo también en ese espacio a generar empoderamiento.

Los relatos de vida y las herramientas del teatro social nos permitieron poner todo lo anterior a disposición del público y compartir con un gran número de chicas sus dificultades, pero también sus ganas y entusiasmo de encontrar soluciones a los problemas que ellas consideran importantes. Estas herramientas permitieron potenciar los usos y prácticas que permiten desarrollar una relación positiva con las tecnologías y un sentimiento de competencia hacia las mismas, como primer paso para la inclusión.

El tipo de intervención realizado permitió mostrar la problemática a personas ubicadas en diferentes posiciones: padres, madres, educadores, compañeros, chicas interesadas en tecnología, chicas y chicos que no, - pero que pueden apoyar a sus compañeras interesadas-, investigadores e instituciones interesadas en disminuir o acabar con la brecha digital de género, entre otras. El teatro permitió ensayar estrategias diferentes ante situaciones que $a$ priori parecieran superarnos. En ese espacio es posible ensayar, cometer errores, pedir consejo, volver a intentar y buscar conjuntamente nuevas maneras de abordar las dificultades. En el espacio teatral fue posible sentir cómo pequeñas acciones — como dar apoyo a alguien vulnerable - hacen la diferencia. También fue evidente que el hecho de pedir argumentos puede desarmar a quien nos descalifica y que defender la propia decisión no es baladí. Ensayar estas y otras posibilidades deviene fundamental cuando entendemos que la performación del género, (Butler 1998, 2001) y la relación con la tecnología (Wajcman, 2006), se construyen en cada situación concreta y que, por tanto, podemos cambiar esa relación si conseguimos transformar las interacciones que la sostienen.

\section{Devolución a la comunidad}

Si bien definimos que la comunidad objeto de nuestra investigación-acción es, en términos generales, el conjunto de mujeres oprimidas por los procesos de exclusión del dominio de la tecnología, existen al menos tres comunidades más específicas sobre las que se hizo una devolución concreta. En primer lugar, el grupo de chicas entrevistadas, quienes fueron invitadas a ver la obra de teatro creada a partir de sus experiencias. Ellas se reconocieron en las situaciones planteadas en la obra y agradecieron haber podido participar del proyecto y compartir sus experiencias. En segundo lugar, estas experiencias forman hoy parte del sitio web que creamos a raíz del proyecto, a disposición de la comunidad académica y de investigadores preocupados por la brecha digital de género. En general, la página está disponible para todo aquel que quiera corroborar, profundizar o continuar el análisis. En tercer lugar, las chicas y chicos que participaron de la obra pueden ver sus aportes en los vídeos que dispusimos en el sitio web. Cabe mencionar que la obra de teatro sigue presentándose en Cataluña y que, para el 
momento en que finalizamos este artículo, llevaba más de cuarenta representaciones y una decena más programada para los próximos meses.

\section{Conclusiones}

Para poder describir las prácticas y usos de la tecnología asociados a la elección de una ingeniería informática como carrera, buscamos exhaustivamente trayectorias vitales y tecnológicas no-convencionales que, por ser tan distintas a las prácticas usuales de los hombres y a sus accesos a la tecnología, son con frecuencia invisibles. No creemos que estemos frente a un ámbito de hombres ni que las mujeres estén menos interesadas en la tecnología que ellos. Muchas están tan interesadas en la tecnología como muchos hombres, pero es bastante posible que no cultiven su interés gracias a la falta de apoyo para sortear las dificultades. El ámbito de la tecnología es difícil para todos, pero está construido a partir de unas prácticas, valores y estereotipos que excluyen particularmente a las mujeres.

Esta investigación-acción nos permitió mostrar cómo se pueden promover nuevas formas de actuar ante situaciones de opresión o dominio, a través de la creación de una obra de teatro adhoc basada en los datos de una investigación con relatos de vida. En este sentido y teniendo en cuenta los resultados que obtuvimos, las futuras intervenciones en este campo que opten por un proceso o variante de investigación-acción para guiar su desarrollo deben tener como objetivo la disminución de los posibles efectos de discriminación derivados de los usos de las tecnologías. Es importante potenciar la visibilidad de las mujeres en su relación con la tecnología, no solo como usuarias o como personajes secundarios, necesitados de ayuda o rescate. No se trata de hacer tecnología para ellas sino de hacer tecnologías para todas y todos, que no lleve una marca de género, que sea suficientemente retadora para fomentar el interés por la tecnología.

Así pues, consideramos que, en la educación primaria y secundaria, no se deben reproducir las situaciones que hacen incompatible el hecho de ser chica y el de ser experta en tecnología. Para ello, recomendamos profundizar en las estrategias de apoyo que puedan activarse cuando las chicas interesadas en la tecnología sean cuestionadas o invisibilizadas. Integrar una perspectiva de género al uso de las TIC, tanto en sus pedagogías como en sus políticas de acción, permitiría a las jóvenes acceder a ellas con menos dificultad. Del mismo modo, las instituciones de educación superior, así como las facultades y escuelas de ingeniería informática, necesitan desarrollar políticas de captación en las que se visibilice la presencia de las mujeres en el sector y las rutas que ellas siguen, para así tomar distancia de la concepción según la cual las trayectorias masculinas son neutrales o naturales. Esto haría de esos espacios unos donde nadie se sienta agredido por ser lo que es o lo que quiere ser. A nivel social, recomendamos indagar en torno al diseño de programas de intervención para la implementación y fomento del uso de videojuegos que contemplen los intereses de las chicas en los espacios públicos de conexión - p. ej. escuelas y bibliotecas-.

Teniendo en cuenta que cada performación del género es situada y específica, es importante entender cómo y cuándo se discrimina a las mujeres en su relación con la tecnología y seguir investigando las dinámicas de la performación de género. Lo anterior evitaría que la performación de experto en tecnología se aprecie como incompatible con la performación de la feminidad. Si bien lo importante es desestabilizar el binarismo - algo a lo que el estudio de performaciones situadas puede contribuir-, lo más urgente, en este caso, es que las mujeres entren en los ámbitos del dominio masculino a los que no han tenido acceso, lo que, por defecto, ayudará a cuestionar el binarismo dominante.

\section{Referencias}

Bartol, K. M., \& Aspray, W. (2006). The transition of women from academic world to workplace. En J. M. Cohoon \& W. Aspray (Eds.), Women and information technology: Research on under-representation (pp. 377-420). Cambridge: MIT Press.

Beavis, C., \& Charles, C. (2007). Would the 'real' girl gamer please stand up? Gender, LAN cafés and 
the reformulation of the 'girl' gamer. Gender and education, 19(6), 691-705.

Bertaux, D. (2005). Los relatos de vida: perspectiva etnosociológica. Barcelona: Ediciones Bellaterra.

Black, S., Jameson, J., Komoss, R, Meehan, A., \& Numerico, T. (2005): "Women in Computing: A European and International Perspective", En Third European Symposium on Gender and ICT: Working for Change, University of Manchester, United Kingdom. Recuperado de http://ict.open.ac.uk/ gender/papers/black.doc

Boal, A. (2002). Juegos para actores y no actores. Barcelona: Alba Editorial.

Boal, A. (2006). Aesthetics of the oppressed. Oxon: Routledge.

Bonino, L. (1995). Develando los micromachismos en la vida conyugal. En J. Corsi (Ed.) Violencia masculina en la pareja. Una aproximación al diagnóstico y los modelos de intervención (pp. 191-208). Buenos Aires: Paidós.

Burrelli, J. (2008). Thirty-three years of women in SEEE faculty positions (NSF Report 08-308). Recuperado de http://www.nsf.gov/statistics/infbrief/nsf08308/ nsf08308.pdf.

Butler, J. (2001). El género en disputa. El feminismo y la subversión de la identidad. México: Paidós.

Butler, J. (1998). Actos performativos y construcción del género. Debate Feminista, 18, 296-314.

Camp, T., Miller, K., \& Davies, V. (2000). the incredible shrinking pipeline unlikely to reverse. Recuperado de http:/inside.mines.edu/fs_home/tcamp/new-study/ new-study.html.

Carlson, S. (2006). Wanted: female computer scientists. The Chronicle of Higher Education, 52 (19), 35-38.

Castaño, C. (2008). La segunda brecha digital. Madrid: Ediciones Cátedra.

Castaño, C., \& Webster, J. (2011).Understanding women's presence in ICT: The life course perspective. International Journal of Gender, Science and Technology, 3 (2), 364-386.

Cheryan, S., Drury, B. J., \& Vichayapai, M. (2012). Enduring influence of stereotypical computer science role models on women's academic aspirations. Psychology of Women Quarterly, 37(1), 72-79. http:// doi.org/10.1177/0361684312459328
Corneliussen, H. G. (2011). Gender-Technology Relations: Exploring Stability and Change. Nueva York: Palgrave Macmillan.

Dee, H. M., Petrie, K. E., Boyle, R. D., \& Pau, R. (2009). Why are we still here? Experiences of successful women in computing. SIGCSE Bulletin, 41(3), 233-237.

Denzin, N. K., \& Lincoln, Y. S. (2000). Handbook of qualitative research. California: Sage.

Departamento de Ciencia Política y Derecho Público (2013). Maletín de recursos. Género y Ciencia.

Universitat Autònoma de Barcelona. Recuperado de http://158.109.131.198/catedra/images/Publicacions/ Maletin-de-Recursos-sobre-Genero-Ciencia. pdf.

European Union (2013). Women active in the ICT sector. Executive summary. Recuperado de http://bookshop.europa.eu/en/women-active-in-the-ict-sectorpbKK0113433/.

Faulkner, W., \& Lie, M. (2007). Gender in the Information Society: Strategies of Inclusion Gender. Technology and Development, 11(2), 157-177.

Fine, C. (2010). Delusions of gender: How our minds, society, and neurosexism create difference. New York: WW Norton \& Company.

Galpin, V. (2002). Women in computing around the world. ACM SIGCSE Bulletin, 34(2), 94-100.

Gil-Juárez, A., Feliu, J., \& Vitores, A. (2010). Performatividad tecnológica de género: explorando la brecha digital en el mundo del videojuego. Quaderns de Psicologia, 12(2), 209-226. Recuperado de http:// www.quadernsdepsicologia.cat/article/view/758.

Gil-Juárez, A., Feliu, J., Vitores, A., \& Vall-llovera, M. (2011). Expressing and managing emotions in a gendered site: women and technology. En The fourth conference of the Psychosocial Studies Network. Affect, subjectivity and social order/disorder, Centre for Medical Humanities, Brighton.

Gil-Juárez, A., Vitores, A., Feliu, J., \& Vall-llovera, M. (2011b). Brecha digital de género: una revisión y una propuesta. Revista Teoría de la Educación, 12(2), 25-53. Recuperado de http://campus.usal. es/ revistas_trabajo/index.php/revistatesi/article/ view/8272/8276

Gómez Ferri, J. (2004). La profesión de informátic@. Una investigación preliminar sobre la generiza- 
ción de la profesión. En M. J. González, E. Espinar \& M. C. Albert (Coords.), II Congreso Online del Observatorio para la Cibersociedad. Recuperado de http://www.cibersociedad.net/congres2004/grups/fitxacom_publica2.php?idioma $=$ ca\&id $=533 \&$ grup $=48$.

Hayes, C. C. (2010). Computer science. The incredible shrinking woman. En T. J. Misa (Ed.), Gender Codes. Why Women Are Leaving Computing (pp. 25-50). New Jersey: Wiley/IEEE Computer Society Press.

Howard, L. A. (2004). Speaking theatre/doing pedagogy: Re-visiting the Theatre of the Oppressed. Communication Education, 53(3), 217-233.

Heeter, C., Egidio, R., Mishra, P., Winn, B., \& Winn, J. (2008). Alien games: Do girls prefer games designed by girls? Games and Culture, 4(1), 74-100. http://doi.org/10.1177/1555412008325481

Husbands Fealing, K., \& Myers, S. L. (2012). Pathways v. pipelines to broadening participation in the stem workforce. Working Paper Series, January 13. Recuperado de http://papers.ssrn.com/sol3/papers. cfm?abstract_id $=2020504$.

Instituto de la Mujer y para la Igualdad de Oportunidades. (2015). Situación de la e-Igualdad en España. 2012 y 2013. Ministerio de Sanidad, Servicios Sociales e Igualdad. Recuperado de http:// www.inmujer.gob.es/areasTematicas/sociedadInfo/publicaciones/docs/Situacion_e_igualdad_en_ Esp_2012_2013.pdf.

Jenson, J., \& de Castell, S. (2008). Theorizing gender and digital gameplay: Over-sights, accidents and surprises. Eludamos, 2(1), 15-25.

Jenson, Jennifer, \& de Castell, Suzanne (2010). Gender, Simulation, and Gaming: Research Re-view and Redirections. Simulation Gaming, 41, 51-71.

Jesse, J. K. (2006). The poverty of the pipeline metaphor: The AAAS/CPST study of nontraditional Pathways into IT/CS education and the workforce. En J. Cohoon \& W. Aspray (Eds.), Women and Information Technology: Research on underrepresentation (pp. 239-78). Cambridge: MIT Press.

Kafai, Y. B. (1998). Video game designs by children: Consistency and variability of gender differences. En J. Cassell \& H. Jenkins (Eds.), Barbie to Mortal
Kombat: Gender and computer games (pp. 90-114). Cambridge: MIT Press.

Lagesen, V. A. (2008). A cyberfeminist utopia? Perceptions of gender and computer science among malaysian women Computer Science students and faculty. Science, Technology $\mathcal{E}$ Human Values, 33, 5-27.

Leventman, P. G. (2007). Multiple pathways toward gender equity in the United States Information Technology Workforce. En C. J. Burger, E. G. Creamer \& P. S. Meszaros (Eds.), Reconfiguring the firewall: Recruiting women to information technology across cultures and continents (pp. 211-238). Wellesley: AK Peters.

Lockwood, P. (2006). "Someone like me can be successful”: Do college students need same-gender role models? Psychology of Women Quarterly, 30, 36-46.

Margolis, J., \& Fisher, A. (2002). Unlocking the clubhouse. Women in computing. Cambridge: MIT Press.

Miller, R. (Comp.) (2004). Biographical Research Methods, Vol I, II, III, IV. London: Sage.

Montenegro, M. (Coord.) (2011). Intervenció social: Controvèrsies teòriques i metodològiques. Barcelona: Editorial UOC.

Pérez-Fuentes, P. y Andino, S. (2003). Las desigualdades de género en el sistema público universitario vasco. Vitoria-Gasteiz : Emakunde, Instituto Vasco de la Mujer.

Puga Rayo, I. (2012). Teatro del Oprimido: dispositivo crítico para la Psicología Social Comunitaria. Sociedad y Equidad, 3, 195-210.

Pujadas, J. J. (1992). El método biográfico: El uso de las historias de vida en Ciencias Sociales. Madrid: CIS.

Rubin, H. J., \& Rubin, I. S. (1995). Qualitative interviewing: The art of hearing data. Thousand Oaks: Sage.

Sanz, V. (2005). Women's careers in Computer Engineering. Case study: Technical University of Madrid. En Third European Symposium on Gender and ICT: Working for Change, University of Manchester, United Kingdom. Recuperado de http:// ict.open.ac.uk/gender/papers/ sanz.doc

Sanz, V. (2008). Mujeres e ingeniería informática: el caso de la Facultad de Informática de la UPM. Arbor, 184(733), 905-915. 
Schutt, R. K. (2001). Investigating the Social World. Thousand Oaks: Pine Forge Press.

Soe, L., \& Yakura, E. K. (2008). What's wrong with the pipeline? Assumptions about gender and culture in IT work. Women's Studies, 37(3), 176-201.

Strauss, A., \& Corbin, J. (1998). Basics of qualitative research: Techniques and procedures for developing grounded theory ( $2^{\mathrm{a}}$ ed.). California: Sage.

Unidad de Mujeres y Ciencia. (2014). Científicas en cifras 2013. Ministerio de Economía y competitividad. Secretaría de estado de investigación, desarrollo e Innovación. Recuperado de http://www.idi.mineco.gob.es/stfls/MICINN/Ministerio/FICHEROS/ UMYC/Cientificas_cifras_2013.pdf.

Vergés, N. (2012). Gender and ICT: The self-inclusion process of women in ICT. An aproach from artistic technologists and computer technologists (Tesis Doctoral). Universitat Oberta de Catalunya, Barcelona. Vergés, N., Cruells, E., \& Hache, A. (2009). Retos y potencialidades para las mujeres en la participación del desarrollo de la sociedad de la información. Feminismo/s, 14, 163-182. Recuperado de http:// www.donestech.net/files/feminismos14donestech. doc.

Vigdor, L. (2011). A techno-passion that is not one: Rethinking marginality, exclusion, and difference. International Journal of Gender, Science and Technology, 3(1). Recuperado de http://genderandset. open.ac.uk/

Vitores, A., \& Gil-Juárez, A. (2015). The trouble with 'women in computing': a critical examination of the deployment of research on the gender gap in computer science. Journal of Gender Studies. Publicación anticipada en línea. http://dx.doi.org/10 $.1080 / 09589236.2015 .1087309$

Wajcman, J. (1991). Feminism Confronts Technology. Cambridge: Polity Press.

Wajcman, J. (2006). El Tecnofeminismo. Madrid: Cátedra.

West, C., \& Zimmerman, D. H. (1987). Doing gender. Gender $\mathcal{F}$ Society, 1, 125-151. 
